\title{
Mavacamten for hypertrophic cardiomyopathy: effectiveness and value
}

\author{
A summary from the Institute for Clinical and Economic Review's California \\ Technology Assessment Forum
}

Molly Beinfeld, MPH; Jason H Wasfy, MD, MPhil; Surrey Walton, PhD, MA; Jyotirmoy Sarker, MPharm, MBA, MBiotech; Emily Nhan; David M Rind, MD, MSc; and Steven D Pearson, MD, MSc

Hypertrophic cardiomyopathy (HCM) is a genetic disorder involving sarcomeres in heart muscle that can cause symptoms such as chest discomfort and shortness of breath, particularly with exertion. Apart from managing symptoms, key components of therapy include placement of implanted cardioverterdefibrillators for patients at high risk of sudden death and anticoagulation for patients who have both HCM and atrial fibrillation. ${ }^{1}$

For patients with a specific subtype of HCM, hypertrophic obstructive cardiomyopathy (HOCM), heart muscle issues cause obstruction of the left ventricular outflow tract (LVOT). For some patients with HOCM, this obstruction can be an important contributor to exertional symptoms. For HOCM patients with shortness of breath related to LVOT obstruction, medications can improve symptoms. Beta blockers and calcium channel blockers reduce the forcefulness of the heart's contraction, decreasing the LVOT gradient, which then improves symptoms. However, beta blockers and calcium channel blockers have important side effects, including fatigue that can interfere with work or daily activities, dizziness, and sexual dysfunction.

When these first-line therapies are insufficient or not well tolerated, second-line treatment options include adding disopyramide or performing septal reduction procedures. ${ }^{1}$ Disopyramide has important side effects as well, and drug shortages have significantly limited access to the long-acting version. Septal reduction procedures include surgical myectomy (a type of open-heart surgery) or alcohol septal ablation, a percutaneous procedure that reduces the thickness of the heart muscle in the area causing LVOT obstruction. These procedures can have substantial benefit, but outcomes may be better at centers of excellence, and they have a low-but nonzero-risk of complications, including death. ${ }^{2}$ Given these considerations, there remains substantial unmet need for new options in the care of patients with symptomatic HOCM, particularly among patients who do not have good access to specialized centers.

Mavacamten, a novel agent for symptomatic HOCM, is under review by the US Food and Drug Administration, with a decision to approve the treatment expected in April 2022. Mavacamten reduces adenosine triphosphatase activity in cardiac myosin heavy chain, one of the proteins in heart muscle cells, and reduces the contraction of the heart that can contribute to obstruction. ${ }^{3}$ The Institute for Clinical and Economic Review (ICER) conducted a systematic literature review and cost-effectiveness analysis to evaluate health and

\section{Author affiliations \\ Molly Beinfeld, MPH; Emily Nhan; David M Rind, MD, MSc; and Steven D Pearson, MD, $\mathrm{MSc}$, Institute for Clinical and Economic Review, Boston, MA. Jason H Wasfy, MD, MPhil, Massachusetts General Hospital and Harvard Medical School, Boston, MA. Surrey Walton, PhD, MA, and Jyotirmoy Sarker, MPharm, MBA, MBiotech, University of Illinois at Chicago College of Pharmacy, Chicago, IL.}

AUTHOR CORRESPONDENCE:

Molly Beinfeld, mbeinfeld@icer.org

J Manag Care Spec Pharm 2022;28(3):369-75

Copyright $\odot 2022$, Academy of Managed Care Pharmacy. All rights reserved.

economic outcomes of mavacamten in patients with symptomatic HOCM. Evaluations were also conducted of the available evidence on septal reduction procedures and on treatment with disopyramide. Complete details of ICER's systematic literature search and protocol, as well as the methodology and model structure for the economic evaluation, are available on ICER's website at https://icer.org/ hypertrophic-cardiomyopathy-2021/. Here, we present the summary of 
our findings and highlights of the policy discussion with key stakeholders held at a public meeting of the California Technology Assessment Forum (CTAF) on October 22, 2021.

\section{Summary of Findings}

\section{MAVACAMTEN}

The systematic literature review on mavacamten identified 1 phase 3 randomized clinical trial with open-label extension and 1 open-label phase 2 trial. For disopyramide, only retrospective studies were available, and for septal reduction procedures, we used existing systematic reviews that incorporated meta analyses. Our review also evaluated information from conference abstracts and data submitted by the manufacturer.

The pivotal trial of mavacamten, EXPLORER-HCM, was a multicenter 30 -week phase 3 trial that randomized 251 patients with HOCM in a 1:1 ratio to 5-15 mg of oral mavacamten or placebo. ${ }^{4}$ Patients were eligible to participate if they were aged 18 years or older, met the criteria for HOCM based on current clinical guidelines, and had documented left ventricular ejection fraction (LVEF) of at least $55 \%$ and New York Heart Association (NYHA) class II-III symptoms. Patients were excluded if they were on current treatment with disopyramide or had been treated with septal reduction therapy (myectomy or septal ablation) within 6 months before screening. ${ }^{5}$

Participants in the EXPLORER-HCM trial had a mean age of 58.5 years and were predominantly male and White (91\%). In the mavacamten group, 9\% of participants had undergone septal reduction therapy; all but $3 \%$ were taking background medication (beta blockers and/or calcium channel blockers); and 72\% were classified as having NYHA class II symptoms at baseline. ${ }^{4}$ The EXPLORER trial also included a 5-year extension study (MAVA-LTE) to evaluate the long-term safety and efficacy of mavacamten. At the time of the ICER report, only interim results from MAVALTE were available. ${ }^{6}$

The primary outcome in the EXPLORER trial was "clinical response" defined in 1 of 2 ways: (1) at least $1.5 \mathrm{~mL} / \mathrm{kg}$ per min increase in pressure of oxygen in venous blood $\left(\mathrm{pVO}_{2}\right)$ and at least 1 NYHA class reduction or (2) at least $3.0 \mathrm{~mL} / \mathrm{kg}$ per min increase in $\mathrm{pVO}_{2}$ and no worsening of NYHA class. ${ }^{5}$ Clinical response was achieved by 45 (37\%) patients in the mavacamten arm at 30 weeks compared with $22(17 \%)$ in the placebo arm $(\mathrm{P}=0.0005) .{ }^{4}$ Thirty-two $(27 \%)$ patients in the mavacamten group achieved NYHA class I status with LVOT peak gradients less than $30 \mathrm{~mm} \mathrm{Hg}$ at 30 weeks compared with 1 patient (1\%) in the placebo group, a difference of $26.6 \%$ (95\% CI $=18.3 \%-34.8 \%)$.
In addition to the primary outcome, improvements in function and health-related quality of life were assessed using the Kansas City Cardiomyopathy Questionnaire (KCCQ), a validated heart failure-specific instrument. ${ }^{7}$ Greater improvements in multiple KCCQ domains, including quality of life, were observed for patients in the mavacamten group compared with the placebo group at 30 weeks. $^{8}$ KCCQ improvements were all greater than the minimal clinically important difference, estimated to be between 4 and 6 points across all domains. ${ }^{7}$ All improvements in KCCQ reversed to baseline 8 weeks after withdrawal of treatment during the washout period. ${ }^{8}$

Treatment-emergent side adverse events are common in patients with HOCM, and in the EXPLORER trial, $88 \%$ of participants in the mavacamten group reported any adverse event compared with $79 \%$ in the placebo arm. ${ }^{4}$ Common adverse events included ventricular tachycardia, atrial fibrillation, palpitations, cardiac failure, and angina. Eleven serious adverse events were reported by $10(8 \%)$ patients in the mavacamten group vs 20 serious events reported by 11 (9\%) in the placebo group. Serious adverse events leading to discontinuation were reported by $1.6 \%$ of participants in the mavacamten group vs $0.8 \%$ in the placebo arm.

Given the mechanism of action of mavacamten, the study protocol required temporary treatment discontinuation for LVEF less than 50\%, excessive QT interval, or mavacamten plasma concentration of more than $1,000 \mathrm{ng} / \mathrm{mL}^{5}$ During the study period, 3 patients on mavacamten and 2 patients on placebo temporarily discontinued treatment due to LVEF decreases, and an additional 4 patients on mavacamten had LVEF less than $50 \%$ at week $30 .{ }^{4}$ In 3 of these 4 patients, the LVEF returned to normal, and in 1 patient, severe systolic dysfunction developed after an atrial fibrillation ablation with complications. One of the patients in the mavacamten group had a procedural complication after ablation for atrial fibrillation and severe LVEF decrease but partially recovered to LVEF 50\% during the washout period.

\section{DISOPYRAMIDE}

In the retrospective study of disopyramide, 40 (34\%) of 118 patients receiving the drug required a major intervention (myectomy, septal ablation, or dual-chamber pacing) because of inadequate symptom control, persistent gradients, or drug intolerance. Interventions were required a mean (SD) of 2.0 (2.1) years after initiating disopyramide. ${ }^{9}$ Among patients who did not require an intervention and remained on treatment during the study period, mean (SD) peak flow gradient decreased from 75 (33) $\mathrm{mm}$ Hg at baseline to 40 (32) $\mathrm{mm} \mathrm{Hg}(\mathrm{P}<0.0001)$, and mean (SD) NYHA class declined from $2.3(0.7)$ to $1.7(0.6 ; \mathrm{P}<0.0001$; Table 1$)$. 
Key Results of Randomized Controlled Trial of Mavacamten, Retrospective Study of Disopyramide, and Systematic Review of Septal Reduction Therapies

\begin{tabular}{|c|c|c|c|c|c|c|c|}
\hline & & \multicolumn{2}{|c|}{$\begin{array}{l}\text { Randomized controlled trial of } \\
\text { mavacamten (EXPLORER) }\end{array}$} & \multicolumn{2}{|c|}{$\begin{array}{c}\text { Retrospective study of } \\
\text { disopyramide (Sherrid 2005') }\end{array}$} & \multicolumn{2}{|c|}{$\begin{array}{c}\text { Systematic review of septal reduction } \\
\text { therapies (Liebregts } 2015^{10} \text { ) }\end{array}$} \\
\hline & & $\begin{array}{l}\text { Mavacamten } \\
(n=123)\end{array}$ & $\begin{array}{l}\text { Placebo } \\
(n=128)\end{array}$ & $\begin{array}{c}\text { Disopyramide } \\
\text { without } \\
\text { intervention } \\
(n=78)\end{array}$ & $\begin{array}{c}\text { Disopyramide } \\
\text { with } \\
\text { intervention } \\
(n=40)\end{array}$ & $\begin{array}{l}\text { Septal ablation } \\
\qquad(n=2,013)\end{array}$ & $\begin{array}{l}\text { Myectomy } \\
(n=2,791)\end{array}$ \\
\hline \multicolumn{2}{|c|}{$\begin{array}{l}\text { LVOT change from baseline, } \\
\text { mean (SD) } \mathrm{mm} \mathrm{Hg}\end{array}$} & $-47 \quad(40)$ & $-10 \quad(30)$ & $-35^{a}$ & $-10^{\mathrm{a}}$ & $\begin{array}{c}-71 \% \\
\text { (IQR: }-67 \text { to }-90)^{\mathrm{b}}\end{array}$ & $\begin{array}{l}-77 \% \\
(\text { IQR: }-69 \text { to }-90)^{\mathrm{b}}\end{array}$ \\
\hline \multicolumn{2}{|c|}{$\begin{array}{l}\text { NYHA class change from } \\
\text { baseline, mean (SD) } \mathrm{mm} \mathrm{Hg}\end{array}$} & NR & NR & $-0.6^{a}$ & 0 & $\begin{array}{c}-45 \% \\
(I Q R:-45 \text { to }-50)^{\mathrm{b}}\end{array}$ & $\begin{array}{c}-45 \% \\
(\text { IQR: }-44 \text { to }-48)^{\mathrm{b}}\end{array}$ \\
\hline \multirow{3}{*}{$\begin{array}{l}\text { NYHA class } \\
\text { distribution at } \\
\text { follow-up, } \\
n(\%)\end{array}$} & Class I & $61(49.6)$ & 27 (21.1) & $29(37.2)^{\mathrm{a}}$ & NR & NR & $N R$ \\
\hline & Class II & $52(42.3)$ & 74 (57.8) & $42(53.8)^{a}$ & NR & NR & NR \\
\hline & Class III/IV & $8 \quad(6.5)$ & 25 (19.5) & $7 \quad(9.0)^{a}$ & NR & $\begin{array}{c}8 \% \\
(\text { IQR: } 8 \text { to } 8)^{b}\end{array}$ & $\begin{array}{c}4.5 \% \\
\text { (IQR: } 4.5 \text { to } 12)^{\mathrm{b}}\end{array}$ \\
\hline
\end{tabular}

andicates a statistically significant difference.

${ }^{b}$ Weighted median.

'Timepoints varied between studies.

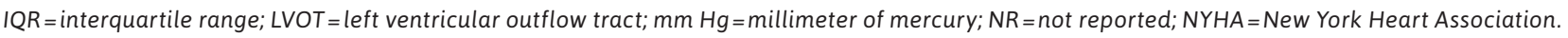

As an antiarrhythmic agent, disopyramide has known risks of proarrythmia. In a single-site retrospective study focusing on the safety of disopyramide, The corrected QT (QTc) interval was prolonged by a mean $19 \mathrm{msec}$ compared with baseline. ${ }^{11}$ The proportion of patients with QTc prolongation of at least $460 \mathrm{msec}$ was $16 \%$ at baseline and 33\% after disopyramide. Although both the (1) magnitude of risk associated with medication-induced QTc prolongation and (2) any QTc cut-off above which medications should be stopped are not well defined, clinicians consider stopping medications above a QTc of $500 \mathrm{msec}^{12}$

\section{SEPTAL REDUCTION PROCEDURES}

In a 2015 systematic review and meta-analysis of septal reduction therapies, the pooled median percentage reduction in NYHA class after both septal ablation and myectomy was $45 \%$, and the median proportion of patients remaining in NYHA class III/IV was only $8 \%$ after septal ablation and $5 \%$ after myectomy $(P=0.43) .{ }^{10}$ Median LVOT gradient reduction was $71 \%$ after septal ablation and $77 \%$ after myectomy $(P=0.63$; Table 1$)$.

The most important safety concern with septal reduction therapies is procedure-related adverse events. In a 2015 systematic with meta-analysis of septal reduction therapies, pooled peri-procedural mortality ( $<30$ days) was $1.3 \%$ in the septal ablation cohorts and $2.5 \%$ in the myectomy cohorts $(\mathrm{P}=0.051) .{ }^{10}$ In-hospital outcomes are worse at lower-volume centers. ${ }^{2}$

\section{LIMITATIONS OF THE CLINICAL EVIDENCE}

Although mavacamten improved physiologic parameters and symptoms in the EXPLORER trial out to 30 weeks, the available data leave important questions about longer-term benefits and potential harms. Clinical experts advising the ICER review differed on whether reductions in ejection fraction with mavacamten reflected beneficial improvements in cardiac function or worrisome changes that could be associated with clinical harm over longer observation times. A meaningful reduction in ejection fraction was also observed in 2 patients in the REDWOOD-HCM phase 2 trial of another myosin inhibitor, aficamten, potentially suggestive of a class effect. ${ }^{13}$ Another general concern about the data on mavacamten is that more than $90 \%$ of patients in the EXPLORER trial were White. It therefore remains unknown whether there are physiological subtypes of HOCM more prevalent within patients of color that lead to differential effects compared with those in White patients.

With disopyramide, while patient groups and some clinical experts have argued for its role as an important later-line medical therapy for HOCM ${ }^{14}$ it has not been studied in high-quality randomized trials, either against placebo or against mavacamten, limiting the ability to make direct or indirect comparisons of the agents. The retrospective analysis of disopyramide that is the primary source of information has several potential sources of bias that could exaggerate the treatment effect of disopyramide, including 


\begin{tabular}{|c|c|c|c|c|c|}
\hline Treatment & Comparator & $\begin{array}{l}\text { Cost per QALY } \\
\text { gained, } \$\end{array}$ & $\begin{array}{l}\text { Cost per life-year } \\
\text { gained, } \mathbf{3}\end{array}$ & $\begin{array}{l}\text { Cost per evLY } \\
\text { gained, } \$\end{array}$ & $\begin{array}{l}\text { Cost per additional } \\
\text { NYHA year, \$ }\end{array}$ \\
\hline \multirow{4}{*}{ lavacamten } & Standard treatment & $1,200,000$ & Undefined & $1,200,000$ & 219,000 \\
\hline & Disopyramide & $1,500,000$ & Undefined & $1,500,000$ & 278,000 \\
\hline & Myectomy & Dominated & $5,600,000$ & $N / A^{b}$ & Dominated \\
\hline & Septal ablation & Dominated & $7,000,000$ & $N / A^{b}$ & Dominated \\
\hline
\end{tabular}

aPrice assumed for mavacamten was a placeholder of $\$ 75,000$ per year.

${ }^{b}$ Incremental cost per evLY gained not applicable due to fewer lifetime QALYs for mavacamten compared with myectomy and septal ablation. eVLY=equal value of life-years; $N / A=$ not applicable; $N Y H A=N e w$ York Heart Association; $Q A L Y=$ quality-adjusted life-year.

treatment selection bias and differential loss to follow-up. Furthermore, there has been a prolonged shortage of longacting disopyramide in the US market, making it difficult for clinicians to consider it as an option for most patients.

There are also important limitations to the evidence on septal reduction procedures. There are no randomized data comparing surgical myectomy to septal ablation or comparing either type of septal reduction therapy to mavacamten. An ongoing randomized trial is examining whether mavacamten can reduce the need for septal reduction procedures ${ }^{15}$ but this does not directly assess the relative benefits of mavacamten compared with these procedures. Furthermore, it is unclear whether the trial results of septal procedures performed at academic centers of excellence can be generalized to other centers.

\section{LONG-TERM COST-EFFECTIVENESS}

We developed a de novo semi-Markov decision analytic model for the evaluation of mavacamten used along with first-line standard of care for patients with symptomatic HOCM. The model focused on an intention-to-treat analysis, with a hypothetical cohort of patients with HOCM starting the model and different pathways representing different treatment options. The treatment pathways of the model included mavacampten with standard first-line therapy, standard first-line therapy alone, and myectomy, septal ablation, or disopyramide each including standard first-line therapy. The model cycle length was 4 weeks to best fit with the available clinical data. The model used a lifetime time horizon, and costs and outcomes were discounted at 3\% per year. Full details on ICER's cost-effectiveness analysis and model are available on ICER's website at https://icer.org/ hypertrophic-cardiomyopathy-2021/.
Treatment effects were based on changes in NYHA class after treatment. Focusing on changes in NYHA class was viewed as the most valid approach to translate study results across all treatment options into improvements in quality of life that could be directly compared. Literature-based estimates of NYHA class changes for myectomy, septal ablation, and for disopyramide were extrapolated to be comparable with those generated by the patient population in the EXPLORER trial. Based on discussions with clinical experts and a literature review, mortality rates were assumed constant across NYHA class in this model. The proportion of alive patients across NYHA class was assumed to be constant after cycle 8 (week 32) in the mavacamten and standard first-line therapy only arms, whereas constant mortality effects were assigned past cycle 1 (week 4) in the myectomy, septal ablation, and disopyramide arms. As such, the only mortality effect across treatments in the base-case model was associated with perioperative mortality from myectomy and septal ablation, and the findings of the model were therefore identical whether health gains were summarized with quality-adjusted life-years (QALYs) or equal value of life-years gained (evLYG).

A placeholder price of $\$ 75,000$ per year was used for mavacamten based on industry analyst commentary. ${ }^{16}$ Using this placeholder price, model results showed mavacamten producing more QALYs than standard first-line therapy but with very high additional costs. This resulted in an incremental cost per QALY of approximately $\$ 1,200,000$, far above standard cost-effectiveness thresholds (Table 2). The incremental cost per QALY is even higher when comparing mavacamten with disopyramide. In contrast, when compared with myectomy and septal ablation, mavacamten costs more and produces fewer QALYs. In cost-effectiveness parlance, the septal reduction procedures "dominate" mavacamten. Full results are available on ICER's website at https://icer.org/hypertrophic-cardiomyopathy-2021/. 


\section{TABLE 3 Votes on Other Contextual Considerations for Mavacamten}

\begin{tabular}{|c|c|c|c|c|c|}
\hline Contextual consideration & $\begin{array}{l}\text { Very low } \\
\text { priority }\end{array}$ & Low priority & $\begin{array}{l}\text { Average } \\
\text { priority }\end{array}$ & High priority & $\begin{array}{l}\text { Very high } \\
\text { priority }\end{array}$ \\
\hline $\begin{array}{l}\text { Acuity of need for treatment of individual patients based on the short-term } \\
\text { risk of death or progression to permanent disability }\end{array}$ & 2 & 3 & 7 & 3 & 0 \\
\hline $\begin{array}{l}\text { Magnitude of the lifetime impact on individual patients of the condition } \\
\text { being treated }\end{array}$ & 0 & 0 & 4 & 10 & 1 \\
\hline
\end{tabular}

\section{LIMITATIONS OF THE COST-EFFECTIVENESS MODEL}

There were only 30 weeks of data available for mavacamten in the EXPLORER trial on which to base projected treatment effects by NYHA class, and as noted earlier, the EXPLORER data may not generalize to other patient populations. The treatment effects are based solely on changes in NYHA class, since that was the only common measure usable across the various treatment arms but which may miss relevant improvements related to treatments within NYHA classes. Further, the utilities in the model across NYHA class come from patients in the trial and may not generalize to other HOCM patients. In addition, the evidence for myectomy, septal ablation, and disopyramide comes from less rigorous observational studies, while the evidence for mavacamten used in the modeling came from the EXPLORER trial.

Although we felt it reasonable to model multiple comparators together, we had insufficient data to conduct a network meta-analysis or other quantitative analyses to control more precisely for differences in baseline characteristics of patients. Not discussed in this article are results from hypothetical scenarios to evaluate the potential broader societal perspective in our cost-effectiveness analysis. Data did not exist to support a specific societal perspective, but we included several scenarios based on hypothetical effects on productivity and employment opportunity that, even under extreme assumptions, found mavacamten to be far above commonly cited cost-effectiveness thresholds at its placeholder price.

\section{Policy Discussion}

The CTAF is one of the independent appraisal committees convened by ICER to engage in the public deliberation of the evidence on clinical and cost-effectiveness of health care interventions. The CTAF is composed of medical evidence epxerts, including clinicians, methodologists, and patient advocates. The ICER report on mavacamten for symptomatic HOCM was the subject of a CTAF meeting on October 22 , 2021. Following the discussion, CTAF panel members deliberated on key questions raised by ICER's report. For the population of patients with symptomatic HOCM, the panel voted as follows: (1) 6-9 that currently available evidence is adequate to demonstrate that the net health benefit of mavacamten added to background therapy is superior to that provided by background therapy alone and (2) 3-10 that currently available evidence is adequate to demonstrate that the net health benefit of mavacamten is superior to that provided by disopyramide.

The CTAF also voted on "potential other benefits" and "contextual considerations" offered by mavacamten to the individual patient, caregivers, the delivery system, other patients, or the public that was not available in the evidence base nor could be adequately estimated within the costeffectiveness model. The results of these votes are shown in Tables 3 and 4 . Value votes were not taken at the CTAF Public Meeting because a net price for mavacamten was not available.

Following the discussion of the evidence, a policy roundtable was convened to deliberate on how best to apply the evidence to the use of mavacamten for symptomatic HOCM. The policy roundtable members included 2 patient advocates, 2 clinical experts, and 2 payers. The manufacturer declined to send a representative to participate in the policy roundtable. The discussion reflected multiple perspectives and opinions; therefore, none of the statements below should be taken as a consensus view held by all participants. The full set of policy recommendations can be found in the Final Evidence Report on the ICER website: https://icer.org/hypertrophic-cardiomyopathy-2021/

Select key policy recommendations for mavacament for HOCM are as follows:

Recommendation 1: All stakeholders have a responsibility to facilitate meaningful patient access to multidisciplinary centers of excellence for HOCM in ways that do not exacerbate disparities. Access to the expertise offered by these experienced multidisciplinary centers is critically important. Ideal care pathways could include regular care from an accessible local cardiologist with intermittent input from experts at centers of excellence. 


\section{TABLE 4 Votes on Other Potential Benefits or Disadvantages for Mavacamten}

\begin{tabular}{|c|c|c|c|c|c|}
\hline Potential other benefit or disadvantage & $\begin{array}{c}\text { Major } \\
\text { negative } \\
\text { effect }\end{array}$ & $\begin{array}{c}\text { Minor } \\
\text { negative } \\
\text { effect }\end{array}$ & $\begin{array}{l}\text { No } \\
\text { difference }\end{array}$ & $\begin{array}{c}\text { Minor } \\
\text { positive } \\
\text { effect }\end{array}$ & $\begin{array}{c}\text { Major } \\
\text { positive } \\
\text { effect }\end{array}$ \\
\hline $\begin{array}{l}\text { Patients' ability to achieve major life goals related to education, work, or } \\
\text { family life }\end{array}$ & 0 & 1 & 1 & 11 & 2 \\
\hline $\begin{array}{l}\text { Caregivers' quality of life and/or ability to achieve major life goals related } \\
\text { to education, work, or family life }\end{array}$ & 0 & 1 & 2 & 11 & 1 \\
\hline Society's goal of reducing health inequities & 0 & 1 & 8 & 5 & 1 \\
\hline Opportunity to improve access to treatment & 0 & 0 & 6 & 8 & 1 \\
\hline $\begin{array}{l}\text { Availability of a treatment with different timing and types of risks and } \\
\text { benefits, relative to existing procedural and surgical options }\end{array}$ & 0 & 0 & 1 & 9 & 5 \\
\hline
\end{tabular}

Recommendation 2: Payers should provide adequate reimbursement for telemedicine and interprofessional consultation between centers of excellence and community cardiologists to facilitate both access to care and appropriate subspecialist expertise when needed. If payers restrict access to mavacamten to providers at specialized centers of excellence, they should work with the patient community as well as clinical experts to select these centers.

Recommendation 3: It is reasonable for payers to require an attempt to manage symptomatic HOCM with beta blockers and calcium channel blockers before approving mavacamten; however, intolerable side effects or contraindications are reasonable justifications for defining treatment failure of beta blockers and/or calcium channel blockers.

Recommendation 4: It is unreasonable for insurers to require either myectomy or septal ablation before approval of mavacamten. The decision to initiate an oral medication vs undergoing a procedure or surgery is very dependent on the preferences and circumstances of individual patients. Shared decision making is appropriate in these situations.
Recommendation 5: Unless patients have better access to long-acting disopyramide, it seems unreasonable to consider requiring a trial of disopyramide before coverage of mavacamten. Further clinical evidence on the clinical benefits of disopyramide is needed to strengthen any consideration of this step therapy option.

Recommendation 6: Clinical specialty societies should work with patient organizations to develop and validate standards for centers of excellence for HOCM and work with payers, regulators, and patients to develop educational tools to improve knowledge about the management of HCM among community providers. Furthermore, they should continue to educate cardiologists about the critical importance of shared decision making for all treatment options for HCM.

Recommendation 7: The manufacturer of mavacamten should commit to sponsoring research that will address the lack of evidence on the comparative effectiveness of mavacamten vs disopyramide and septal reduction procedures. The manufacturer of mavacamten should also align the price of mavacamten with transparent estimates of its treatment benefits for patients and families. Pricing should also be moderated to reflect the uncertainty about longer-term safety until such time as further outcomes data are generated.

Recommendation 8: Postapproval clinical registries should be established to detect rare side effects as well as to assess the efficacy of mavacamten in more diverse populations. Since the MAVA-LTE study uses the same population as the EXPLORER trial, there is very limited representation among patients of color. Furthermore, this cohort, which includes 224 patients, will be underpowered to detect rarer side effects among all patients.

\section{DISCLOSURES}

Funding for this summary was contributed by Arnold Ventures, The Donaghue Foundation, Harvard Pilgrim Health Care, and Kaiser Foundation Health Plan to the Institute for Clinical and Economic Review (ICER), an independent organization that evaluates the evidence on the value of health care interventions.

ICER's annual policy summit is supported by dues from AbbVie, America's Health Insurance Plans, Anthem, Alnylam, AstraZeneca, Biogen, Blue Shield of CA, Boehringer-Ingelheim, Cambia Health Services, CVS, Editas, Evolve Pharmacy, Express Scripts, Genentech/Roche, GlaxoSmithKline, Harvard Pilgrim, Health Care Service Corporation, HealthFirst, Health Partners, Humana, Johnson \& Johnson (Janssen), Kaiser Permanente, LEO 
Pharma, Mallinckrodt, Merck, Novartis, National Pharmaceutical Council, Pfizer, Premera, Prime Therapeutics, Regeneron, Sanofi, Sun Life Financial, uniQure, and United Healthcare.

Beinfeld, Nhan, Rind, and Pearson are employed by ICER. Through their affiliated institutions, Wasfy, Walton, and Sarker received funding from ICER for the work described in this summary. Walton also reports consulting fees from Second City Outcomes Research. Wasfy reports personal fees from Biotronik and Pfizer; grants from National Institutes of Health, National Football League Players Association and American Heart Association; and travel support from American College of Cardiology. Sarker has nothing additional to disclose.

\section{ACKNOWLEDGMENTS}

The authors thank Mel Wittington, Laura Cianciolo, Sue Kwon, Mrinmayee Joshi, and Maggie Houle for their contributions to this report.

\section{REFERENCES}

1. Ommen SR, Mital S, Burke MA, et al. 2020 AHA/ACC Guideline for the Diagnosis and Treatment of Patients With Hypertrophic Cardiomyopathy: Executive Summary. Circulation. 2020;142(25):e533-57.

2. Kim LK, Swaminathan RV, Looser P, et al. Hospital volume outcomes after septal myectomy and alcohol septal ablation for treatment of obstructive hypertrophic cardiomyopathy: US Nationwide Inpatient Database, 2003-2011. JAMA Cardiology. 2016;1(3):324-32.

3. Green EM, Wakimoto H, Anderson RL, et al. A small-molecule inhibitor of sarcomere contractility suppresses hypertrophic cardiomyopathy in mice. Science. 2016;351(6273):617-21.
4. Olivotto I, Oreziak A, Barriales-Villa R, et al. Mavacamten for treatment of symptomatic obstructive hypertrophic cardiomyopathy (EXPLORER-HCM): a randomised, double-blind, placebocontrolled, phase 3 trial. Lancet. 2020;396(10253):759-69.

5. Ho CY, Olivotto I, Jacoby D, et al. Study design and rationale of EXPLORER-HCM: evaluation of mavacamten in adults with symptomatic obstructive hypertrophic cardiomyopathy. Circ Heart Fail. 2020;13(6):e006853.

6. Rader F, Choudhury L, Saberi S, et al. Long-term safety of mavacamten in patients with obstructive hypertrophic cardiomyopathy: interim results of the Mava-Long Term Extension (LTE) study. J Am Coll Cardiol. 2021;77(18):532.

7. Butler J, Khan MS, Mori C, et al. Minimal clinically important difference in quality of life scores for patients with heart failure and reduced ejection fraction. Eur J Heart Fail. 2020;22(6):999-1005.

8. Spertus JA, Fine JT, Elliott P, et al. Mavacamten for treatment of symptomatic obstructive hypertrophic cardiomyopathy (EXPLORER-HCM): health status analysis of a randomised, doubleblind, placebo-controlled, phase 3 trial. Lancet. 2021;397(10293):2467-75.

9. Sherrid MV, Barac I, McKenna WJ, et al. Multicenter study of the efficacy and safety of disopyramide in obstructive hypertrophic cardiomyopathy. J Am Coll Cardiol. 2005;45(8):1251-58.

10. Liebregts M, Vriesendorp PA, Mahmoodi BK, Schinkel AFL, Michels M, ten Berg JM. A systematic review and meta-analysis of long-term outcomes after septal reduction therapy in patients with hypertrophic cardiomyopathy. JACC Heart Fail. 2015;3(11):896-905.
11. Adler A, Fourey D, Weissler-Snir A, et al. Safety of outpatient initiation of disopyramide for obstructive hypertrophic cardiomyopathy patients. J Am Heart Assoc. 2017;6(6):e005152.

12. Al-Khatib SM, LaPointe NM, Kramer JM, Califf RM. What clinicians should know about the QT interval. JAMA. 2003;289(16):2120-27.

13. Cytokinetics. Sarcomere directed therapies. 2021. Accessed January 20, 2022. https://cytokinetics.com/ wp-content/uploads/2021/07/2021-Corporate-Presentation-General-072821.pdf

14. Maron MS, Ommen SR. Exploring new and old therapies for obstructive hypertrophic cardiomyopathy: mavacamten in perspective. Circulation. 2021;143(12):1181-83.

15. Desai MY, Wolski K, Owens A, et al. Study design and rationale of VALORHCM: evaluation of mavacamten in adults with symptomatic obstructive hypertrophic cardiomyopathy who are eligible for septal reduction therapy. Am Heart J. 2021;239:80-89.

16. Bell J. MyoKardia pops as heart drug passes most important test. BioPharma Dive Brief. May 11, 2020. Accessed December 10, 2021. https:// www.biopharmadive.com/news/ myokarida-explorer-mavacamten-studyresults/577678/ 